\title{
Ibrutinib/Bendamustine/Rituximab Regimen
}

National Cancer Institute

\section{Source}

National Cancer Institute. Ibrutinib/Bendamustine/Rituximab Regimen. NCI Thesaurus.

Code C160548.

A regimen consisting of ibrutinib, bendamustine and rituximab that may be used in the treatment of certain types of relapsed or refractory chronic lymphocytic leukemia (CLL)/small lymphocytic lymphoma (SLL). 